\title{
One-relator groups with torsion are coherent
}

\author{
Larsen Louder and Henry Wilton*
}

August 11, 2020

\begin{abstract}
We show that any one-relator group $G=F /\langle w\rangle\rangle$ with torsion is coherent - i.e., that every finitely generated subgroup of $G$ is finitely presented - answering a 1974 question of Baumslag in this case.
\end{abstract}

\section{Introduction}

Definition 1.1. A group $G$ is coherent if every finitely generated subgroup of $G$ is finitely presentable.

A well known question of Baumslag asks whether every one-relator group $F /\langle\langle w\rangle$ is coherent Bau74, p. 76]. It is a curious feature of one-relator groups that the case with torsion, in which the relator $w$ is a proper power, is often better behaved than the general case; most famously, one-relator groups with torsion are always hyperbolic New68, and Wise proved that one-relator groups with torsion are residually finite, indeed linear Wis12. In this note we answer Baumslag's question affirmatively for one-relator groups with torsion.

Theorem 1.2. If $G$ is a one-relator group with torsion - that is, $G \cong F /\left\langle w^{n}\right\rangle$, for $n>1$ - then $G$ is coherent.

In 2003, Wise circulated Wis03, including a purported proof of the following conjecture, stated as Theorem 4.13 of that paper.

Conjecture 1.3. If $X$ is a compact 2-complex with non-positive immersions then $\pi_{1} X$ is coherent.

The reader is referred to, for instance, [LW17] or [HW16] for the definition of non-positive immersions. On his webpage, Wise acknowledges that there is a gap (found by Mladen Bestvina) in the proof of [Wis03, Theorem 4.13]. In Wis05, Wise used Conjecture 1.3 (stated as Theorem 1.5 of that paper) in a proof that Theorem 1.2 follows from the Strengthened Hannah Neumann Conjecture. The latter conjecture has more recently been proved independently by Friedman [Fri15] and Mineyev Min12.

*Supported by EPSRC Standard Grant EP/L026481/1. 
In summary, the results of Wis05] are conditional on Conjecture 1.3, which remains open, and therefore Theorem 1.2 was not hitherto known unconditionally. After our proof was circulated, we learned from Wise that he has also given an unconditional proof of Theorem 1.2 in Wis18, a revised version of Wis03.

Our proof (and also that of Wis18) uses Wise's w-cycles conjecture (Theorem 3.11), which was proved independently by the authors [LW17] and by HelferWise [HW16].

The outline of the proof is as follows. We realize $G$ as the fundamental group of a compact, aspherical orbicomplex $X$. Since one-relator groups are virtually torsion-free, there is a finite-sheeted covering map $X_{0} \rightarrow X$ so that $G_{0}=\pi_{1} X_{0}$ is torsion free. We then use the $w$-cycles conjecture to show that, whenever $Y \leftrightarrow X_{0}$ is an immersion from a compact two-complex without free faces, the number of two-cells in $Y$ is bounded by the number of generators of $\pi_{1}(Y)$. In the final step, a folding argument expresses an arbitrary finitely generated subgroup $H$ of $G_{0}$ as a direct limit of fundamental groups of 2-complexes with boundedly many 2 -cells, and we deduce that $H$ is finitely presented.

\section{Acknowledgements}

Thanks to Jim Howie and Hamish Short for pointing out an error in Section 4 of an earlier version.

\section{One-relator orbicomplexes}

Let $F$ be a finitely generated free group, and $G=F /\left\langle w^{n}\right\rangle$ a one-relator group, where $w \in F$ is not a proper power. In the usual way, $F$ can be realized as the fundamental group of some finite topological graph $\Gamma$, and $w$ by a continuous map $w: S^{1} \rightarrow \Gamma$. (Since we are only interested in $w$ up to conjugacy, we ignore base points.) Let $D_{n} \subseteq \mathbb{C}$ be the closed unit disk equipped with a cone point of order $n$ at the origin. The orbicomplex

$$
X=\Gamma \cup_{w} D_{n}
$$

provides a natural model for $G$, in the sense that $G$ is the (orbifold) fundamental group of $X$. We call $X$ a one-relator orbicomplex. (There is a much more general theory of orbicomplexes - see, for instance, Hae91 or [BH99, Chapter III.C] but the one-relator orbicomplexes defined here are sufficient for our purposes.) When $n=1, X$ is a one-relator complex.

A map of 2-complexes is a morphism if it sends $n$-cells homeomorphically to $n$-cells, for $n=0,1,2$. A morphism of 2-complexes $Y \rightarrow Z$ is an immersion if it is a local injection; in this case, we write $Y \rightarrow Z$. If $Y$ is a 2-complex and $X$ is the one-relator orbicomplex defined above, a continuous map $Y \rightarrow X$ is a morphism if it sends vertices to vertices, edges homeomorphically to edges, and restricts, on each 2-cell, to the standard degree- $n$ map $p_{n}: D_{1} \rightarrow D_{n}$ given by $p_{n}(z)=z^{n}$. A morphism $Y \rightarrow X$ is an immersion if it is locally injective away from the cone points in the 2-cells (again, we write $Y \rightarrow X$ ), and a covering if 
it is locally a homeomorphism except at the cone points. The next definition plays a crucial role in our argument.

Definition 2.1 (Degree). If $f: Y \rightarrow X$ is an immersion of two-dimensional (orbi)complexes, then the degree of $f$, denoted by $\operatorname{deg} f$, is the minimum number of preimages of a generic point in a 2-cell of $X$. That is: if $X$ is a 2-complex, then $\operatorname{deg} f$ is the minimum number of preimages of any point in the interior of a 2-cell of $X$; and if $X$ is a one-relator orbicomplex with 2-cell $D_{n}$, then $\operatorname{deg} f$ is the number of preimages of any point in the interior of $D_{n}$ except 0 .

Every one-relator group is virtually torsion free [FKS72, and it follows that the orbi-complex $X$ is finitely covered by a genuine 2 -complex. This can be seen using the covering theory for complexes of groups developed in [BH99, but we give a low-tech proof below.

Theorem 2.2 (Unwrapped covers of one-relator complexes). Let $G=F /\left\langle w^{n}\right\rangle$ be a one-relator group with $w$ not a proper power, and $X$ the orbicomplex defined above. Then there is a finite-sheeted covering map

$$
X_{0} \rightarrow X
$$

where $X_{0}$ is a compact, connected 2-complex.

Proof. Let $X^{\prime}$ be $\Gamma \cup_{w^{n}} D$, the (genuine) two-complex that is the result of gluing a disc $D$ to the graph $\Gamma$ along the map $w^{n}$. Note that there is a natural morphism $X^{\prime} \rightarrow X$ that induces an isomorphism of fundamental groups. Let $G_{0}$ be a torsion-free subgroup of finite index in $G$, and let $X_{0}^{\prime} \rightarrow X^{\prime}$ be the corresponding covering space. Since $w$ has order $n$ in $G$ [MKS04, Corollary 4.11], the 2-cells of $X_{0}^{\prime}$ come in families of cardinality $n$, such that all the 2-cells in each family have the same attaching map. Let $X_{0}$ be the quotient of $X_{0}^{\prime}$ obtained by collapsing each family to a single 2-cell. Picking one 2-cell from each family specifies an inclusion $X_{0} \hookrightarrow X_{0}^{\prime}$, and the quotient map $X_{0}^{\prime} \rightarrow X_{0}$ is then visibly a retraction, and an isomorphism on fundamental groups by the Seifert-van Kampen theorem. The composition

$$
X_{0} \hookrightarrow X_{0}^{\prime} \hookrightarrow X^{\prime} \rightarrow X
$$

is the required covering map.

We emphasize that the complex $X_{0}$ in the above theorem is a 2-complex, not just an orbicomplex. That is, the covering map $X_{0} \rightarrow X$ restricts to $p_{n}$ on each 2-cell. We will call such a cover $X_{0}$ unwrapped. 


\section{A bound on the number of 2-cells}

A two-complex $Y$ is reducible if it has a free face 1 Writing

$$
\partial_{Y}: \coprod S^{1} \rightarrow Y^{(1)}
$$

for the disjoint union of the attaching maps of the 2-cells, this means that there is an edge $e$ of the 1-skeleton $Y^{(1)}$ such that $\partial_{Y}^{-1}(e)$ consists of a single edge in $\coprod S^{1}$. If $e$ is such an edge and $Y^{\prime}$ is the 2-complex obtained by collapsing the face of $Y$ incident at $e$, then the natural inclusion map $Y^{\prime} \hookrightarrow Y$ is a homotopy equivalence, and induces an isomorphism on fundamental groups. Of course, if $Y$ is not reducible it is called irreducible.

The main theorem of [LW17] (or [HW16]) can be restated as a result about immersions to one-relator orbicomplexes, as follows.

Theorem 3.1. Let $X$ be a one-relator orbicomplex, $Y$ a finite 2-complex and $f: Y \leftrightarrow X$ an immersion. If $Y$ is irreducible then $\chi\left(Y^{(1)}\right)+\operatorname{deg} f \leq 0$.

Proof. This follows from [LW17, Theorem 1.2], with $\Gamma=X^{(1)}, \Gamma^{\prime}=Y^{(1)}, \rho$ the restriction of $f$ to $\Gamma^{\prime}, \Lambda=w$, and $\mathbb{S}$ the disjoint union of the boundaries of the 2-cells of $Y$. If some edge of $Y^{(1)}$ is not hit by a 2-cell, then we may remove that edge, increasing $\chi\left(Y^{(1)}\right)$. Otherwise, $Y$ is reducible in the sense of [LW17], so [LW17, Theorem 1.2] applies, taking $\Lambda^{\prime}=\partial_{Y}$ and $\sigma$ the induced map from the boundaries of the 2-cells of $Y$ to the boundary of the 2-cell of $X$.

Here, we apply Theorem 3.1 to relate the number of 2-cells of $Y$ to the rank of its fundamental group. (By the rank of a group, we mean the minimal cardinality of a generated set.)

Corollary 3.2. Let $f: Y \leftrightarrow X$ be an immersion from a finite, irreducible 2complex $Y$ to a one-relator orbicomplex $X$. Then

$$
\chi(Y)+(n-1) \mid\{2 \text {-cells in } Y\} \mid \leq 0 .
$$

In particular,

$$
\mid\{2 \text {-cells in } Y\} \mid \leq \frac{\operatorname{rk}\left(\pi_{1}(Y)\right)-1}{n-1}<\operatorname{rk}\left(\pi_{1}(Y)\right)
$$

if $n>1$.

Proof. By Theorem 3.1, $\chi\left(Y^{(1)}\right)+\operatorname{deg}(f) \leq 0$. Since $f$ restricts to $p_{n}$ on each 2-cell of $Y$, it follows that

$$
\operatorname{deg} f=n \mid\{2 \text {-cells in } Y\} \mid
$$

\footnotetext{
${ }^{1}$ Note that this definition is slightly stronger than the definition given in [LW17, where a 2-complex was called 'reducible' if it has a free face or a free edge. The definition given here is more convenient in this context, since the complexes $Y_{i}$ produced by Lemma 4.4 are irreducible in this sense, but not in the sense of [LW17].
} 
since $X$ is one-relator.

The Euler characteristic of $Y$ is the Euler characteristic of $Y^{(1)}$ plus the number of two-cells in $Y$, so Theorem 3.1 implies the first assertion. The second assertion now follows from the first, using the trivial fact that

$$
1-\operatorname{rk}\left(\pi_{1} Y\right) \leq \chi(Y)
$$

for any connected 2-complex $Y$.

In the case with torsion, Corollary 3.2 gives a bound on the the number of 2-cells of an immersion in terms of the rank of the fundamental group. In order to make a connection to arbitrary finitely generated subgroups of $G$, we use folding, in the spirit of Stallings.

\section{Folding}

Folding was introduced by Stallings to study free groups and their subgroups. The next lemma extends [Sta83, Algorithm 5.4] to the context of 2-complexes and their morphisms.

Lemma 4.1. Any morphism of finite 2-complexes $A \rightarrow B$ factors as

$$
A \rightarrow C \mapsto B
$$

where $A \rightarrow C$ is surjective and $\pi_{1}$-surjective. Furthermore, if $A \rightarrow B$ factors as

$$
A \rightarrow D \rightarrow B
$$

then there is a unique immersion $C \rightarrow D$ so that the following diagram commutes.

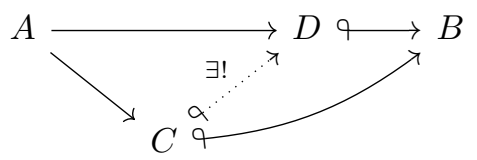

Proof. Folding shows that the map of 1-skeleta factors as

$$
A^{(1)} \rightarrow C^{(1)} \rightarrow B^{(1)}
$$

where $A^{(1)} \rightarrow C^{(1)}$ is surjective and $\pi_{1}$-surjective. We now construct $C$ by pushing the attaching maps of the 2-cells of $A$ forward to $C^{(1)}$ and identifying any 2-cells with the same image in $B$ and equal attaching maps. The resulting map $A \rightarrow C$ is surjective and $\pi_{1}$-surjective. We next check that the natural map $C \rightarrow B$ is an immersion.

Since $C \rightarrow B$ is a morphism, it can only fail to be locally injective at a point $p \in C$ if two higher-dimensional cells incident at $p$ have the same image in $B$. The map of 1-skeleta is an immersion, so this can only occur if two 2-cells $c_{1}, c_{2}$ in $C$, incident at $p$, have the same image in $B$. Because the attaching maps 
of $c_{1}$ and $c_{2}$ agree at $p$ and $C^{(1)} \rightarrow B^{(1)}$ is an immersion, it follows that the attaching maps of $c_{1}$ and $c_{2}$ agree everywhere. Therefore, $c_{1}$ and $c_{2}$ are equal in $C$ by construction.

It remains to prove the universal property. This fact is standard for graphs, which defines the required immersion of 1-skeleta $C^{(1)} \rightarrow D^{(1)}$ uniquely. Let $c$ be a 2-cell in $C$ and let $a_{1}, a_{2}$ be preimages of $c$ in $A$. By construction, $a_{1}$ and $a_{2}$ have the same boundary in $C^{(1)}$ and the same image in $B$. Therefore, their images $d_{1}, d_{2}$ respectively in $D$ have the same boundary in $D^{(1)}$ and the same image in $B$. But $D \leftrightarrow B$ is an immersion, and it follows that $d_{1}=d_{2}$. Therefore, we may extend the map $C^{(1)} \rightarrow D^{(1)}$ across $c$ in a unique way, as required.

A free edge of a 2-complex is an edge of the 1-skeleton that is not in the image of the attaching map of any 2-cell. The next result appeals to the Scott lemma (which plays a crucial role in the proof of coherence for 3-manifold groups) to represent a finitely generated, freely indecomposable subgroup by an immersion from a compact 2-complex without free edges or faces.

Lemma 4.2. Let $X$ be a 2-complex, $G=\pi_{1}(X)$, and $H$ a non-trivial, finitely generated, freely indecomposable subgroup of $G$. There is an immersion from a compact irreducible 2-complex without free edges $f: Y \leftrightarrow X$ such that $f_{*} \pi_{1} Y$ is conjugate to $H$.

Proof. By the Scott lemma Sco73. Lemma 2.2], there is a surjection from a finitely presented group $H^{\prime} \rightarrow H$ that does not factor through a free product. Since $H^{\prime}$ is finitely presented, there is a morphism of combinatorial 2-complexes $Z^{\prime} \rightarrow X$ that represents the composition $H^{\prime} \rightarrow H \rightarrow G$. By Lemma 4.1, this morphism factors as

$$
Z^{\prime} \rightarrow Z \leftrightarrow X
$$

where $Z^{\prime} \rightarrow Z$ is $\pi_{1}$-surjective. By the conclusion of the Scott lemma, $\pi_{1} Z$ is freely indecomposable. Let $Y^{\prime} \subseteq Z$ be a deformation retract of $Z$ obtained by iteratively collapsing free faces. Since $\pi_{1} Y^{\prime}=\pi_{1} Z$ is freely indecomposable, any free edges of $Y^{\prime}$ are separating, and one complementary component of each free edge is simply connected. Let $Y$ be the unique non-simply-connected component obtained from $Y^{\prime}$ by deleting free edges, and let $f: Y \leftrightarrow X$ be the natural immersion. Then $Y$ is as required: $f_{*} \pi_{1} Y$ is conjugate to $f_{*} \pi_{1} Y^{\prime}=H$, and $Y$ has neither free faces nor free edges.

The next two lemmas show that a finitely generated subgroup can be represented by a direct limit of immersions of irreducible 2-complexes. We start with the freely indecomposable case.

Lemma 4.3. Let $X$ be a 2-complex, $G=\pi_{1}(X)$, and $H \leq G$ a finitely generated, freely indecomposable subgroup. Then there is a sequence of $\pi_{1}$-surjective immersions of compact, connected two-complexes

$$
Y_{0} \leftrightarrow Y_{1} \leftrightarrow \cdots \leftrightarrow Y_{i} \leftrightarrow \cdots X
$$

with the following properties. 
(i) Each $Y_{i}$ is irreducible.

(ii) $H=\lim _{1} \pi_{1} Y_{i}$

(iii) The number of free edges of $Y_{i}$ is uniformly bounded.

Proof. If $H$ is trivial then so is the result. Otherwise, let $f: Y \rightarrow X$ be the immersion guaranteed by Lemma 4.2, so that $f_{*} \pi_{1} Y$ is conjugate to $H$. Let $f_{0}: Y_{0} \rightarrow X$ be a wedge of $Y$ with an interval $I$ so that, fixing a basepoint at the end of the interval, $f_{0 *} \pi_{1} Y_{0}=H$. Let $\left(r_{i}\right)$ be an enumeration of representatives of the conjugacy classes of ker $f_{0 *}$, where each $r_{i}$ is an immersed combinatorial loop in the 1-skeleton of $Y \subseteq Y_{0}$.

We now construct the immersions $Y_{i} \rightarrow Y_{i+1}$ inductively, assuming that $\left\{r_{0}, \ldots, r_{i}\right\}$ represent elements of the kernel of $\pi_{1} Y_{i} \rightarrow G$. Let $E_{i+1} \rightarrow X$ be a reduced van Kampen diagram for $r_{i+1}$, and let $Z=Y_{i} \cup_{r_{i+1}} E_{i+1}$. Since $r_{i+1}$ did not cross any free edges of $Y_{i}, Z$ does not have any free faces. We now apply Lemma 4.1 to the natural map $Z \rightarrow X$, which yields

$$
Z \rightarrow Y_{i+1} \leftrightarrow \text { } X
$$

for the desired 2-complex $Y_{i+1}$. Next, we prove properties (i), (ii) and (iii).

By construction, $Y_{0}$ has no free faces. Therefore, we may prove (i) by induction: assuming that $Y_{i}$ has no free faces, we claim that $Y_{i+1}$ has no free faces. Suppose by way of contradiction that an edge $e$ is a free face of a 2-cell $c$ in $Y_{i+1}$. Since $Y_{i}$ immerses into $Y_{i+1}$, and $Y_{i}$ has no free faces, it follows that $e$ is not in the image of $Y_{i}$. Therefore, $e$ is the image of an edge $e^{\prime}$ in the interior of $E_{i+1}$. The two neighbouring 2-cells of $E_{i+1}$ both map to $c$ folding across $e^{\prime}$, contradicting the hypothesis that $E_{i+1}$ is reduced.

Property (ii) is immediate by construction. For (iii), simply note that any free edges of $Y_{i}$ are the image of an edge in the interval $I$.

Finally, we deal with possibly freely decomposable subgroups, by appealing to the Grushko decomposition.

Lemma 4.4. Let $X$ be a 2-complex, $G=\pi_{1}(X)$, and $H \leq G$ a finitely generated subgroup. Then there is a sequence of $\pi_{1}$-surjective immersions of compact, connected two-complexes

$$
Y_{0} \leftrightarrow Y_{1} \rightarrow \cdots \leftrightarrow Y_{i} \leftrightarrow \cdots X
$$

with the following properties.

(i) Each $Y_{i}$ is irreducible.

(ii) $H=\lim _{1} \pi_{1} Y_{i}$

(iii) The number of edges of $Y_{i}$ that are not incident at a 2-cell is uniformly bounded. 
Proof. Let $H=H_{0} * \cdots * H_{p} * F_{q}$ be the Grushko decomposition of $H$. For each $1 \leq r \leq p$, let

$$
Y_{0}^{r} \leftrightarrow Y_{1}^{r} \leftrightarrow \cdots \leftrightarrow Y_{i}^{r} \leftrightarrow \rightarrow \cdots X
$$

be the sequence provided by applying Lemma 4.3 to $H_{r}$. Let $Z \rightarrow X$ be a graph immersed in the 1-skeleton with $\pi_{1} Z=F_{q}$. For each $i$, let

$$
Y_{i}^{\prime}=Y_{i}^{1} \vee \cdots \vee Y_{i}^{p} \vee Z
$$

and let $Y_{i} \rightarrow X$ be the immersion obtained by applying Lemma 4.1 to $Y_{i}^{\prime}$.

The required immersion $Y_{i} \rightarrow Y_{i+1}$ exists by the universal property of Lemma 4.1; see the following commutative diagram.

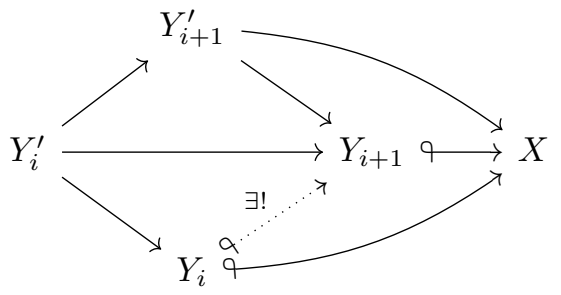

Properties (i), (ii) and (iii) are clear from the construction.

We are now ready to prove our main result.

Proof of Theorem 1.2. Realize $G$ as the fundamental group of a one-relator orbicomplex $X$. Let $H$ be a finitely generated subgroup of $G$. Let $G_{0} \leq G$ be a torsion-free subgroup of finite index, corresponding to the unwrapped cover $X_{0} \rightarrow X$ provided by Theorem 2.2. Since a finite extension of a finitely presented group is finitely presented, we may replace $H$ by $H \cap G_{0}$, and so assume that $H \leq G_{0}$. Consider the sequence of immersions

$$
Y_{0} \leftrightarrow Y_{1} \leftrightarrow \cdots \leftrightarrow Y_{i} \leftrightarrow \cdots X_{0}
$$

provided by Lemma 4.4 taking $X_{0}$ for $X$. By Corollary 3.2 the number of 2-cells of each $Y_{i}$ is bounded. Each 2-cell of $Y_{i}$ is a copy of the unique 2-cell of $X$, hence has boundary of bounded length. Combining this with item (iii) of Lemma 4.4, we see that the number of 1-cells (and hence also 0-cells) of $Y_{i}$ is also bounded. Since $X_{0}$ is finite, there are only finitely many combinatorial types of immersions $Y_{i} \rightarrow X_{0}$. Because $Y_{i} \rightarrow X_{0}$ factors through $Y_{i+1} \rightarrow X_{0}$, there is an infinite subsequence

$$
Y_{i_{1}} \rightarrow Y_{i_{2}} \rightarrow \cdots \uparrow Y_{i_{j}} \rightarrow \cdots X_{0}
$$

so that each map $Y_{i_{j}} \rightarrow Y_{i_{j+1}}$ is a homeomorphism; therefore, $H=\underline{\lim } \pi_{1} Y_{i_{j}}=$ $\pi_{1}\left(Y_{i_{1}}\right)$ is finitely presented, as required. 


\section{$5 \quad$ Groups with good stackings}

The results of [LW17] apply equally well to a class of groups which is rather larger than the class of one-relator groups.

Definition 5.1 (Stacking). Let $X$ be a 2-dimensional orbicomplex and let

$$
\Lambda: \coprod S^{1} \rightarrow X^{(1)}
$$

be the coproduct of the attaching maps of the 2-cells. A stacking of $X$ is a lift of $\Lambda$ to an embedding

$$
\widehat{\Lambda}: \mathbb{S} \equiv \coprod S^{1} \hookrightarrow X^{(1)} \times \mathbb{R}
$$

write $\widehat{\Lambda}(x)=(\Lambda(x), h(x))$. A stacking is called good if, for each component $S$ of the domain of $\Lambda$, there is a point $a \in S$ so that $h(a) \geq h(x)$ for all $x \in \mathbb{S}$ with $\Lambda(a)=\Lambda(x)$, and there is also a point $b \in S$ so that so that $h(b) \leq h(x)$ for all $x \in \mathbb{S}$ with $\Lambda(b)=\Lambda(x)$.

The results of [LW17] apply to the fundamental groups of orbicomplexes with good stackings.

Definition 5.2. We say that a group has a good stacking if it is the fundamental group of a compact, 2-dimensional orbicomplex that admits a good stacking. We say it has a good branched stacking if it has a good stacking, and every 2-cell has a cone point of index at least 2 .

Every one-relator group admits a good stacking [LW17, Lemma 3.4], which is branched if the group has torsion. Corollary 3.2 applies to groups with a good branched stacking. The proof of Theorem 1.2 applies verbatim to groups with good branched stackings, except that groups with good branched stackings are not known to admit unwrapped covers - that is, the analogue of Theorem 2.2 is unknown.

However, we conjecture that Wise's proof that one-relator groups with torsion are residually finite goes through for groups with branched good stackings.

Conjecture 5.3. If $G$ has a good branched stacking then $G$ is hyperbolic, and has a virtual quasiconvex hierarchy, in the sense of [Wis12].

By the results of [Wis12, Conjecture 5.3 would imply that every such group is virtually torsion-free, and hence coherent by the same proof as Theorem 1.2 .

\section{References}

[Bau74] Gilbert Baumslag, Some problems on one-relator groups, Proceedings of the Second International Conference on the Theory of Groups (Australian Nat. Univ., Canberra, 1973) (Berlin), Lecture Notes in Math., vol. 372, Springer, 1974, pp. $75-81$. 
[BH99] Martin R. Bridson and André Haefliger, Metric spaces of non-positive curvature, Grundlehren der Mathematischen Wissenschaften [Fundamental Principles of Mathematical Sciences], vol. 319, SpringerVerlag, Berlin, 1999.

[FKS72] J. Fischer, A. Karrass, and D. Solitar, On one-relator groups having elements of finite order, Proc. Amer. Math. Soc. 33 (1972), 297-301. MR 0311780

[Fri15] Joel Friedman, Sheaves on graphs, their homological invariants, and a proof of the Hanna Neumann conjecture: with an appendix by Warren Dicks, Mem. Amer. Math. Soc. 233 (2015), no. 1100, xii+106, With an appendix by Warren Dicks. MR 3289057

[Hae91] André Haefliger, Complexes of groups and orbihedra, Group theory from a geometrical viewpoint (Trieste, 1990), World Sci. Publ., River Edge, NJ, 1991, pp. 504-540. MR 1170375

[HW16] Joseph Helfer and Daniel T. Wise, Counting cycles in labeled graphs: the nonpositive immersion property for one-relator groups, Int. Math. Res. Not. IMRN (2016), no. 9, 2813-2827.

[LW17] Larsen Louder and Henry Wilton, Stackings and the W-cycles Conjecture, Canad. Math. Bull. 60 (2017), no. 3, 604-612.

[Min12] Igor Mineyev, Submultiplicativity and the Hanna Neumann conjecture, Ann. of Math. (2) 175 (2012), no. 1, 393-414. MR 2874647

[MKS04] Wilhelm Magnus, Abraham Karrass, and Donald Solitar, Combinatorial group theory, second ed., Dover Publications, Inc., Mineola, NY, 2004, Presentations of groups in terms of generators and relations. MR 2109550

[New68] B. B. Newman, Some results on one-relator groups, Bull. Amer. Math. Soc. 74 (1968), 568-571.

[Sco73] G. P. Scott, Finitely generated 3-manifold groups are finitely presented, Journal of the London Mathematical Society. Second Series 6 (1973), 437-440.

[Sta83] John R. Stallings, Topology of finite graphs, Invent. Math. 71 (1983), no. $3,551-565$.

[Wis03] Daniel T. Wise, Coherence, local-indicability and non-positive immersions, Preprint, http://www.gidon.com/dani/tl.cgi?athe=pspapers/NonPositiveCoherence.ps 2003.

[Wis05] Daniel T. Wise, The coherence of one-relator groups with torsion and the Hanna Neumann conjecture, Bull. London Math. Soc. 37 (2005), no. 5, 697-705. MR 2164831 
[Wis12] D. T. Wise, The structure of groups with a quasi-convex hierarchy, Preprint, http://goo.gl/3ctNvX, April 2012.

[Wis18] Daniel T. Wise, Coherence, local-indicability and non-positive immersions, Personal communication, 2018.

L. Louder, Department of Mathematics, University College London, Gower Street, LONDON WC1E 6BT, UK

E-mail address: 1.louder@ucl.ac.uk

H. Wilton, DPMms, Centre for Mathematical Sciences, Wilberforce Road, CamBRIDGE CB3 0WB, UK

E-mail address: h.wilton@maths.cam.ac.uk 